\title{
COSTO DE CAPITAL EN ENTORNOS ECONÓMICOS CAMBIANTES: CASO VALLE DE ABURRÁ (ANTIOQUIA)*
}

\author{
JENNY MOSCOSO ESCOBAR***, CLAUDIA INÉS SEPÚLVEDA RIVILLAS ${ }^{* * * *}$, \\ ANDERSON GARCÍA CANO****** \& AMANDA LUCÍA RESTREPO LONDOÑO \\ UNIVERSIDAD DE ANTIOQUIA
}

Recibido/ Received/ Recebido: 13/03/2012 - Aceptado/ Accepted / Aprovado: 17/07/2012

\begin{abstract}
Resumen
El objetivo del presente estudio es establecer en qué medida el costo de capital de las empresas medianas y grandes pertenecientes al cluster construcción en el Valle de Aburrá, presenta un comportamiento diferente en épocas de auge y de crisis económica. Se utilizó una metodología descriptiva que permite confrontar la teoría financiera con la información empresarial, obtenida del Sistema de Información y Riesgo Empresarial -SIREM- de la Superintendencia de Sociedades de Colombia para el periodo comprendido entre 2005 y 2009. A partir de esta información se calcula el costo de la deuda, el costo del patrimonio y el costo de capital, con el fin de analizar su comportamiento en el período de tiempo considerado. Como principales hallazgos de la investigación, se evidencia que no existe una diferencia marcada en el comportamiento en el costo de capital en los ciclos económicos considerados, hay un crecimiento moderado del patrimonio, siendo similar el comportamiento del costo de capital al del patrimonio.
\end{abstract}

Palabras clave: Costo de capital, Cluster de construcción, Ciclos económicos.

\section{CAPITAL COST IN CHANGING ECONOMIC ENVIRONMENTS: CASE OF VALLE DE ABURRÁ (ANTIOQUIA)}

\begin{abstract}
The objective of this study is to establish in what extent the capital cost of middle and largecompanies that belong to a cluster of construction in Valle de Aburrá, presents a different behavior in boom times than in economic crisis. A descriptive methodology was used, which allows comparing financial theory with enterprise information, from theSuperintendencia de Sociedades de Colombia for the period between 2005 and 2009. From this information the debt cost, patrimony cost and capital cost were calculated, with the purpose of analyzing its behavior during the period of study. Main findings includethat there is not an important difference in the behavior of capital costs in theeconomic cycles considered, there is a moderate growth of patrimony similar to the behavior of capital cost.

Keywords: Capital cost, Construction cluster, Economic cycles.
\end{abstract}

* Artículo derivado de investigación. Fuente Financiadora: Facultad de Ciencias Económicas, Universidad de Antioquia.

** Magister en Finanzas, Universidad Eafit. Estudiante Doctorado Ingeniería, Industria y Organizaciones, Universidad Nacional de Colombia. Docente Universidad de Antioquia, Facultad de Ciencias Económicas - Departamento de Ciencias Administrativas. Correo electrónico: jmoscoso@economicas.udea.edu.co

**** $\quad$ Magister en Finanzas, Universidad Eafit. Docente Universidad de Antioquia, Facultad de Ciencias Económicas - Departamento de Ciencias Administrativas. Correo electrónico: csepulveda@economicas.udea.edu.co

**:*** Administrador de Empresas, Universidad de Antioquia. Profesional Universitario, Secretaría de Educación, Municipio de Medellín, Correo electrónico: ander.garciac@gmail.com

****** Administradora de Empresas, Universidad de Antioquia. Profesional en Formación, Departamento de Sistemas de Información para las Finanzas, EPM. Correo electrónico: restrepolal@economicas.udea.edu.co 


\title{
CUSTO DE CAPITAL EM CONTEXTOS ECONÔMICOS EM MUDANÇA: CASO VALLE DE ABURRÁ (ANTIOQUIA)
}

\begin{abstract}
Resumo
O objetivo do presente estudo é estabelecer até que ponto o custo de capital das empresas médias e grandes pertencentes ao cluster construção no Vale de Aburrá apresenta um comportamento diferente em épocas de auge e de crise econômica. Utilizou-se uma metodologia descritiva que permite confrontar a teoria financeira com a informação empresarial obtida do Sistema de Informação e Risco Empresarial - SIREM - da Superintendência de Sociedades da Colômbia, no período entre 2005 e 2009. A partir desta informação se calcula o custo da dívida, o custo do patrimônio e o custo de capital, com o fim de analisar seu comportamento no período de tempo considerado. Como principais conclusões da pesquisa, se evidencia que não existe uma diferença relevante no comportamento do custo de capital nos ciclos econômicos considerado, e que há um crescimento moderado do patrimônio, sendo similar o comportamento do custo de capital ao do patrimônio.

Palavras chave: Custo de Capital, Cluster de Construção, Ciclos Econômicos.
\end{abstract}

Moscoso, J., Sepúlveda, C., García, A. \& Restrepo, A. (2012) Costo de capital en entornos económicos cambiantes: caso Valle de Aburrá (Antioquia). En: Revista de la Facultad de Ciencias Económicas de la Universidad Militar Nueva Granada. rev.fac.cienc.econ, XX (2).

JEL: G10, M10.

\section{Introducción}

El costo de capital es un valor cuantitativo que busca expresar el costo de los recursos invertidos en la empresa ya sean provenientes de los acreedores o propietarios, y servir de indicador para los accionistas y grupos de interés para la toma de decisiones de inversión; sin embargo, algunas definiciones tienen conceptos errados sobre la interpretación del costo de capital. Una de estas concepciones erróneas es la visión de que es un costo histórico, desconociendo el hecho de que éste corresponde principalmente al desempeño esperado en el futuro sobre los costos de sus componentes en el largo plazo y no el resultado del desempeño histórico.

Por tal razón se considera importante investigar sobre el comportamiento del costo de capital en entornos cambiantes entre los años 2005 y 2009 en el ámbito colombiano; planteado en el presente documento que está organizado en cinco secciones incluyendo ésta introducción. En la segunda sección se encuentran los fundamentos teóricos y los conceptos académicos en los que se basa la investigación, complementado con la sección tres que presenta la metodología definitiva del cálculo del costo de capital para las empresas objeto de estudio. La sección cuatro contiene los resultados y las discusiones y la sección cinco incluye las conclusiones.

\section{Fundamentos teóricos}

El costo de capital es un concepto asociado al desempeño financiero de largo plazo, el cual considera dos componentes, el primero es la deuda financiera conformada principalmente por obligaciones de corto y largo plazo, cuyo pago de intereses genera una deducción de impuestos; y el segundo es el patrimonio, en donde el costo es referenciado por la tasa esperada por los inversionistas.

El concepto de costo de capital, si bien parece un concepto claro, tiene concepciones y puntos de vista en la literatura existente, aunque en general de todas ellas conserva aspectos básicos tales como su estructura y uso, algunas de las definiciones existentes son:

- Rentabilidad Mínima que deben producir los activos de una empresa (García, 2009).

- Es la tasa de interés que los inversionistas, tanto acreedores como propietarios, desean les sea 
pagada para conservar e incrementar sus inversiones en la compañía.

- Costo de la financiación u obtención de fondos por parte de la empresa de diferentes fuentes.

- Tasa de Rendimiento requerida para los proyectos en los que una empresa tiene participación para mantener su valor y atraer fondos (Emery \& Finnerty, 2000).

- Es la tasa de descuento que equipara o iguala el valor presente de los fondos netos y los desembolsos esperados de una empresa.

- Es el costo de oportunidad en el uso de los recursos en la empresa y no en otros proyectos disponibles en el mercado.

- El Costo de Capital no apalancado es la rentabilidad esperada del capital propio de la empresa si ésta se financia enteramente con recursos propios (Grinblatt \& Titman, 2003).

A partir de las anteriores definiciones, se debe reconocer que el costo de capital es un concepto dinámico que se ve afectado por factores económicos del entorno, así como por lineamientos y condicionamientos propios de la empresa; por ello es importante considerar algunos supuestos como el riesgo comercial, el riesgo financiero, los costos de financiación después de impuestos y el tiempo de estimación de las variables. Así mismo "el costo de capital depende principalmente de las aplicaciones de los fondos y no de las fuentes (...) es un error común olvidar este aspecto de importancia fundamental y pensar que el costo de capital de una inversión depende principalmente de dónde y cómo se obtiene el capital" (Ross et al., 2001).

Cabe anotar que si existe una variación con relación al riesgo y que sea de suma importancia en las propuestas de inversión, el costo de capital como tasa de rendimiento requerida para la empresa, en forma global, no resulta apropiada como criterio de decisión.

Es así como este costo se constituye en una herramienta importante para la valoración y la evaluación de proyectos, siendo estos uno de sus principales usos, dado que puede servir como la tasa de descuento usada para la medición del Valor Presente Neto (VPN) de los rendimientos esperados de una alternativa de inversión o de los flujos de caja futuros de la empresa, dado que "el costo de capital se calcula en un momento específico en el futuro. Refleja el costo futuro promedio esperado de los fondos a largo plazo" (Gitman, 2007). Por otra parte también se considera como usos del costo de capital los siguientes:

- Seleccionar alternativas de financiamiento con base al costo mínimo.

- Seleccionar la estructura financiera más adecuada, en el cual el costo de capital se hace mínimo y el valor de la empresa es el máximo.

- Determinar el proceso de generación de valor, utilizándolo para comparar el rendimiento sobre la inversión de capital-ROIC-y el costo de capital.

De ahí que, una de las metodologías para calcular esta herramienta es el costo promedio ponderado de capital -CPPC- el cual se calcula de la siguiente manera:

$$
C P P C=W_{d} * K_{d}(1-T)+W_{p} * K_{p}
$$

Donde, $\mathrm{W}_{\mathrm{d}}$ es el peso de la deuda en la estructura del capital, $\mathrm{W}_{\mathrm{p}}$ el peso del patrimonio, $\mathrm{T}$ la tasa impositiva, $\mathrm{K}_{\mathrm{d}}$ el costo de la deuda antes de impuestos y $\mathrm{K}_{\mathrm{p}}$ el costo del patrimonio.

Es importante anotar que al mencionar la ponderación se está haciendo referencia a la combinación entre las diferentes fuentes de financiación o estructura financiera, que es uno de los temas prioritarios de las finanzas corporativas, más aún en épocas de incertidumbre como la actual. Es por esta razón que las condiciones del entorno, el riesgo del negocio y la estrategia corporativa, son entre otros, factores determinantes para definir la estructura financiera en las empresas.

"La definición de la estructura financiera de la empresa es una de las problemáticas más interesantes del administrador financiero, puesto que implica combinar un conjunto de factores que de una manera independiente o interrelacionada, inciden tanto directa como indirectamente en lograr la mejor composición entre pasivos y patrimonio." (Jaramillo, 2010). 
La estructura financiera de una empresa puede definirse como la combinación de alternativas de financiación entre recursos propios y endeudamiento que determina la posición financiera de la empresa en un momento determinado. La proporción de cada una de las fuentes de financiación depende de varios factores entre ellos el costo de las mismas.

La determinación de esta estructura implica el análisis de los factores relevantes para la empresa, los cuales están asociados a la relación riesgo-rentabilidad, la utilidad, la conformidad, la maniobrabilidad, control, el entorno, las condiciones de la propia empresa y las políticas gubernamentales, los cuales contribuyen a la definición de una estructura financiera "adecuada" de las organizaciones.

Bajo este diseño de cálculo se puede visualizar de mejor manera los factores que afectan al CPPC, como los agentes que se escapan al control de la empresa se contemplan como el nivel de las tasas de interés de la deuda y las tasas tributarias; sin embargo, los factores que la empresa puede controlar son las políticas de la estructura financiera, de dividendos y de inversión, entre otros. Así mismo, se encuentran algunas áreas problemáticas en el costo de capital, los cuáles se deben analizar en cada empresa y sector real de la economía:

- Fondos generados por depreciación.

- Compañías de propiedad privada.

- Problemas de medición.

- Costos de capital en proyectos con varios niveles de riesgo.

- Pesos ponderados de la estructura de capital.

A continuación se presentan los diversos componentes de financiamiento, tanto internos como externos de la empresa; las fuentes internas se originan con las gestiones del patrimonio, particularmente de las utilidades retenidas, por su parte las externas son los pasivos o terceros. Estos son originados bajo distintos mecanismos vía créditos, préstamos, emisiones de acciones o bonos, entre otros.

\subsection{Componente de deuda}

Uno de los componentes principales del costo de capital, está relacionado con la proporción de los acti- vos que está financiada por los acreedores, es decir, la deuda, que puede estar representada por bonos, obligaciones financieras de corto y largo plazo, financiación con proveedores, entre otros. Para efectos de la determinación del costo de capital se considera deuda a la deuda financiera, la cual incluye únicamente aquellos pasivos que de manera explícita contengan una tasa de interés que represente el costo de la deuda antes de impuestos.

Es así como se considera importante iniciar la descripción del componente, a partir de las diferentes definiciones sobre concepto de deuda que han sido abordados desde la normatividad contable colombiana hasta por autores destacados en el medio financiero, como se plantea a continuación:

El Decreto Reglamentario 2650 de 1993 plantea que los pasivos "agrupan el conjunto de las cuentas que representan las obligaciones contraídas por el ente económico en desarrollo del giro ordinario de su actividad, pagaderas en dinero, bienes o en servicios. Comprende las obligaciones financieras, los proveedores, las cuentas por pagar, los impuestos, gravámenes y tasas, las obligaciones laborales, los diferidos, otros pasivos, los pasivos estimados, provisiones, los bonos y papeles comerciales".

Según Vélez (2003) "la deuda es una fuente de financiación que está regida contractualmente. Se pactan los intereses que se pagan y las fechas en que se pagan tanto los intereses como los abonos a capital. El acreedor recibe su dinero, en teoría, sin importarle si la empresa ha recibido beneficios o no. Tiene prioridad sobre los pagos de utilidades o dividendos de los socios o accionistas."

Sin embargo, la literatura tradicional de finanzas propone calcular un promedio ponderado de las diferentes fuentes de financiación para hallar el costo de la empresa antes y después de impuestos, pero éste puede generar distorsiones en la medición del costo de la deuda, por lo que un procedimiento más acertado consiste en tener en cuenta la forma de amortización de cada una de las fuentes de financiación y así conocer para cada período: el saldo, los intereses pagados y como consecuencia el costo combinado, obteniendo por lo tanto una tasa diferente para cada 
período que resulta de la mezcla de las diferentes formas de financiación de la deuda.

Es importante resaltar que el pago de intereses derivado de esta forma de financiación genera contablemente una deducción de impuestos, lo que hace del pasivo una fuente de financiación más barata que cualquier otra. Su forma de cálculo debe incluir el escudo fiscal dado que se presume que la empresa es rentable, de lo contrario no se tendría este beneficio de deducción por impuestos; su cálculo sería:

$$
K_{d}=K(1-t)
$$

Donde $\mathrm{K}_{\mathrm{d}}$ corresponde al costo de la deuda después de impuestos, $\mathrm{K}$ es el costo de la deuda en el mercado y $t$ a la tasa impositiva pagada por cada empresa.

\subsection{Componente de patrimonio}

El Costo del patrimonio puede ser tomado como la tasa que el inversionista espera o requiere como mínima al invertir en determinada empresa; para el cálculo de este costo se pueden utilizar dos metodologías, una es el Modelo de Fijación de Precios de Activos de Capital -CAPM -, consistente en adoptar un modelo de riesgo y retorno. La segunda metodología es un modelo de crecimiento de los dividendos, donde el precio de una acción es el valor presente de sus dividendos que crecen a una tasa constante. Para el caso concreto se encontró que ninguna empresa cotiza en bolsa de valores, por esta razón se procedió a utilizar el modelo CAPM para el cálculo, el cual se describe a continuación.

El modelo CAPM es utilizado para calcular una tasa de descuento en la evaluación y valoración de proyectos y empresas en general, que permite estimar el costo de oportunidad con base en el riesgo sistemático (Villareal, 2005) tomando como principal insumo los siguientes supuestos planteados por Cruz, Villareal, \& Rosillo (2003), Mossin (1966) y Lintner (1965):

- Los inversionistas tienen expectativas homogéneas sobre retornos y varianzas de activos.

- Se puede prestar y pedir prestado a la misma tasa de interés libre de riesgo.
- Los costos de transacción son nulos.

- Los retornos siguen una distribución normal.

- Todos los activos son negociables y divisibles.

- Existe una cantidad amplia de individuos que participan en el mercado.

- Cada individuo debe seleccionar un portafolio que se compondrá de una cantidad determinada de activos riesgosos o libres de riesgo.

- Se tiene una percepción generalizada en el mercado de la distribución de probabilidades del retorno del activo.

- El retorno del portafolio completo es también una variable aleatoria.

Las variables a utilizar en el modelo CAPM son: tasa de retorno libre de riesgo $\left(\mathrm{R}_{\mathrm{f}}\right)$, prima de riesgo $\left(\mathrm{R}_{\mathrm{m}}\right.$ $\mathrm{R}_{\mathrm{f}}$ ) y el coeficiente Beta del activo $(\beta)$.

La prima por riesgo es calculada con base en la información histórica, definida como la diferencia entre los retornos promedio de las acciones y un activo libre de riesgo durante un periodo de medición generalmente entre 5 y 10 años, dado que a mayores periodos de tiempo más se pueden observar las influencias de tendencias marcadas por ciclos económicos.

Para el caso de Colombia, el rendimiento del mercado $\mathrm{R}_{\mathrm{m}}$ se halla con base en el Índice General de la Bolsa de Valores de Colombia IGBC (Vélez, 2003) y la tasa libre de riesgo es considerada igual al retorno del activo que es elegido en el momento de realizar la evaluación; existen dos variantes para realizar la elección de dicha tasa; la primera consiste en seleccionar un papel del tesoro de corto plazo y calcular la prima de riesgo con base en los promedios aritméticos y, la segunda variante, utilizada en este trabajo, toma la tasa de intervención del Banco de la República como tasa libre de riesgo y la prima de riesgo considera las variables descritas anteriormente; así el costo de patrimonio obtenido se utiliza para descontar el flujo de caja de cada año. En cuanto a la variable beta, "mide el grado de co-movimiento entre el retorno del activo y el retorno del portafolio de mercado" (Fernández, 2005). Por tanto, el procedimiento matemático para hallar este componente se deriva de la siguiente fórmula: 


$$
K_{p}=R_{f}+\beta\left(R_{m}-R_{f}\right)
$$

Generalmente, las estimaciones obtenidas para los valores de la beta son enfocadas al mercado estadounidense, sin embargo, la utilización de una beta internacional, reapalancada de acuerdo a la estructura de capital local representa una medida adecuada del riesgo de las industrias para mercados emergentes como el colombiano; ya que existen razones para pensar que valores de las betas más altas o bajas pueden ser causadas por las diferencias existentes entre los mercados de Estados Unidos y de Colombia (Comisión de Regulación de Energía y Gas, 2002).

La beta de una empresa se encuentra determinado por tres factores, el primero el tipo de negocio o clúster donde se encuentra ubicada la empresa, el grado de apalancamiento operacional y el apalancamiento financiero.

La metodología para hallar el valor de la beta es utilizar la covarianza del activo analizado con el mercado y la varianza del riesgo de mercado $\mathrm{R}_{\mathrm{m}}$, así el cálculo de la beta sería:

$$
\beta_{t}=\frac{\operatorname{Cov}\left(R_{t}, R_{m}\right)}{\operatorname{Var}\left(R_{m}\right)}
$$

Teniendo en cuenta lo anterior, la beta utilizada en el cálculo del costo del patrimonio recoge el efecto del apalancamiento financiero, por tal razón es necesario hallar el beta apalancada con la información de las empresas colombianas, la cual se puede encontrar mediante la siguiente relación matemática denominada "fórmula de Hamada" (García, 2003; Damodaran, 2002).

$$
\beta_{L}=\beta_{u}\left[1+\frac{(1-t) \text { Deuda }}{\text { Patrimonio }}\right]
$$

Donde:

$\mathrm{B}_{u}=$ beta de los activos o desapalancada.

$\mathrm{B}_{L}=$ beta de la empresa corregida por apalancamiento financiero o beta apalancada.

$t=\quad$ tasa marginal de impuestos.

Es necesario resaltar que la beta refleja el riesgo sistemático inherente a las operaciones de la empresa, de modo que este riesgo se aumenta con el apalancamiento financiero. Para las empresas que operan en más de un área de negocio, la beta se calcula como el promedio ponderado de las betas de los diferentes sectores, para el cálculo de la beta desapalancada se emplean los valores de mercado de las empresas y en el caso de la apalancada se emplean los valores patrimoniales de mercado. Cuando las empresas no transan en bolsa, entonces se toman como base betas de compañías que transen y resulten comparables (Mejía, 2000).

En concordancia con lo anterior, para calcular este costo para empresas que no cotizan en bolsa, existen otras alternativas adicionales que se describirán a continuación. Vélez (2003), plantea una metodología que consiste en preguntar directamente a los dueños de la compañía su tasa de rentabilidad, sin embargo, ésta no es aplicable para el caso de estudio dado que se cuenta con una base de 291 empresas en Colombia. La segunda alternativa sería aplicar el modelo de Gordon planteado en ecuación (6).

$$
K e=\frac{D}{P}+g
$$

En donde el precio de la acción del mercado (P) se reemplaza por el valor contable del patrimonio, además se utiliza los datos de los dividendos (D) y una tasa de crecimiento (g), pero para el caso de estudio no es aplicable dado que no hay información suficiente de las política de dividendos de las empresas.

\section{Metodología}

La investigación es de tipo descriptivo, es decir busca únicamente describir situaciones; señalando características sobresalientes en la población de estudio correspondientes a una muestra estratificada, seleccionada a criterio, de las empresas medianas y grandes del clúster de construcción del Valle de Aburrá. El trabajo no está interesado en comprobar hipótesis o explicaciones, ni en hacer predicciones, sólo permitirá responder si existe una distancia entre la academia y el sector real al momento de definir el costo de capital.

\subsection{Fuentes de información}

La fuente primaria usada corresponde al Sistema de Información y Riesgo Empresarial -SIREM- de la 
Superintendencia de Sociedades ${ }^{1}$ de Colombia que se compone principalmente de la información de las sociedades colombianas sujetas a vigilancia y control por parte de esta superintendencia.

Basado en lo anterior se construye una base de datos correspondiente a la información de los estados financieros de empresas medianas y grandes con Balance General, Estado de Resultados y Flujo de Caja, de las empresas para los años comprendidos entre 2005 y 2009. Esta base inicial se constituyó con un promedio de 22.420 registros de empresas por año los cuales fueron depurados teniendo como referencia los siguientes criterios:

- Las empresas seleccionadas deben tener como municipio de radicación aquellos que pertenecen al Valle de Aburrá del Departamento de Antioquia: Copacabana, Girardota, Bello, Medellín, Envigado, Itagüí, Sabaneta, La Estrella y Caldas.

- Las empresas deben tener reportes de información continuos para el periodo de referencia 2005-2009, aspecto que se llevo a cabo a través de la comparación año a año del Número de Identificación Tributaria-NIT-.

- Las empresas deben contar con un mismo objeto social durante el periodo de estudio, para este aspecto se tomó como variable proxy el CIIU - Código Industrial Internacional Uniforme- esto con el fin de tomar aquellas empresas que conservarán su código CIIU durante todo el periodo.

A continuación se realizó la clasificación de estas empresas por tamaño tomando como referencia el valor de sus activos según la ley 590 del 2000 modificada por la ley 905 de 2004. De este proceso, el $62 \%$ de las empresas se ubicarón en la clasificación de mediana y grande para el año 2009, luego se realizó una identificación de estas empresas para determinar, a través del código CIIU cuales pertenecen al clúster de construcción teniendo como base la clasificación de la Cámara de Comercio de Medellín para dicho clúster, lo cual permitió obtener un total de 127 empresas con información continua, dentro de este clúster en la base de datos correspondiente a la muestra tomada para la investigación, sin embargo, después de realizar los cálculos respectivos de los indicadores, se encontró que 20 empresas presentan inconsistencias en la información dado que se obtienen resultados atípicos para uno o más años, en cuyo caso éstos fueron eliminados, con lo que el marco final de empresas fue de 107 (69 grandes y 38 medianas) empresas corresponden al clúster de construcción el equivalente a 535 registros analizados. Se toma como muestra el clúster de construcción por ser un sector empresarial del país reconocido en todo el territorio y sus datos consistentes en el periodo de tiempo estudiado.

Es importante resaltar que para el estudio se consideró que el periodo de auge corresponde a los años 2005 - 2006 y la época de crisis considera los años 2007, 2008 y 2009, decisión fundamentada en el comportamiento de variables macroeconómicas como el $\mathrm{PIB}^{2}$ y el Índice General de la Bolsa de Valores de Colombia ${ }^{3}$.

Las fuentes secundarias de información fueron: la revisión bibliográfica y documental, que de cuenta de los resultados relacionados con las empresas seleccionadas, así como estudios académicos que dieron soporte investigativo y conceptual para el adecuado logro de los objetivos.

\subsection{Medición de las variables de los componentes del costo de capital}

En este apartado se describen los cálculos de los componentes más relevantes para la obtención del $\mathrm{CPPC}$ de las empresas estudiadas:

- El cálculo del costo de la deuda, se realizó a través de un proxy de los intereses de la deuda financiera de las empresas analizadas. Su cálculo es el cociente entre los gastos no operacionales

\footnotetext{
Vease: http://www.supersociedades.gov.co/

Véase Banco de la República: http://www.banrep.gov.co/estad/dsbb/seminario.htm

Véase Bolsa de Valores de Colombia: http://www.bvc.com.co/pps/tibco/portalbvc/Home
} 
de la empresa y el promedio del valor de la deuda de corto y largo plazo del año actual y del año anterior.

- La tasa de impuestos usada correspondió a la tasa oficial de impuesto de renta de Colombia para cada año, que se ubica entre un 33 a $35 \%$.

- Para el caso del costo de patrimonio se utilizó el modelo CAPM, en donde se describirá a continuación el manejo para cada una de las variables denotadas en la ecuación (3):

- El Rendimiento del mercado se halla con base en el Índice General de la Bolsa de Valores de Colombia IGBC.

- Se consideró que la tasa libre de riesgo corresponde al valor de la tasa de intervención del Banco de la República de Colombia, siendo esta referente para el mercado colombiano.

- Para el caso de la beta se toma como la desapalancada $\left(\beta_{\mathrm{u}}\right)$ el promedio de los $\beta_{\mathrm{u}}$ de los sectores estadounidenses calculados por Damodaran (2002) que se relacionan en Tabla 1.

Tabla 1. Sectores empresariales Estados Unidos vs. Clúster Valle de Aburrá4

\begin{tabular}{|l|l|}
\hline \multicolumn{1}{|c|}{ Sector } & \multicolumn{1}{c|}{ Clúster } \\
\cline { 1 - 1 } Building Materials & \\
\cline { 1 - 1 } Cement \& Aggregates & \multirow{2}{*}{ Construcción } \\
\cline { 1 - 1 } Heavy Construction & \\
\cline { 1 - 1 } Homebuilding & \\
\hline
\end{tabular}

Otra manera de calcular la beta para el caso colombiano, fue considerar empresas del mismo sector que cotizan en la bolsa de valores y a partir de esta beta encontrar un beta desapalancado con la estructura de financiación y proceder a apalancarlo para la muestra objeto de estudio. Con este beta se procedió a calcular el costo de capital del estudio dado que se tenían empresas de referencia en el país y no era necesario aplicarle ningún factor de prima de riesgo adicional.
El cálculo de la estructura de financiamiento de las empresas del clúster, tomó como referencia la deuda financiera y el patrimonio de las empresas. En relación con la deuda, se incluyeron aquellos rubros que corresponden a la deuda financiera de corto y largo plazo, entre los que se encuentran las obligaciones financieras, cuentas comerciales y obligaciones laborales; en cuanto al patrimonio, se usó el patrimonio bruto dado que este incluye tanto las revalorizaciones como las utilidades de ejercicios anteriores que hacen parte integral de los recursos generados por la empresa y que son de propiedad de los accionistas o dueños.

Por tal razón, para realizar una lectura objetiva de los resultados del costo de capital, se presenta la información de acuerdo a la densidad de la estructura, es decir se dividió la información en 5 quintiles de la siguiente manera:

Tabla 2. Composición de estructura financiera por quintiles $^{5}$

\begin{tabular}{|c|c|c|}
\hline Quintil & $\begin{array}{c}\text { \% Composición } \\
\text { Estructura Patrimonio }\end{array}$ & $\begin{array}{c}\text { \% Composición } \\
\text { Estructura Deuda }\end{array}$ \\
\hline 1 & $100-80$ & $0-20$ \\
\hline 2 & $80-60$ & $20-40$ \\
\hline 3 & $60-40$ & $40-60$ \\
\hline 4 & $40-20$ & $60-80$ \\
\hline 5 & $20-0$ & $80-100$ \\
\hline
\end{tabular}

\section{Resultados y discusión}

\subsection{Análisis de datos}

"El Clúster Construcción se define como la concentración geográfica en Medellín y Antioquia, de empresas e instituciones especializadas y complementarias en la actividad de la construcción de edificaciones e infraestructura; las cuales interactúan entre sí, creando un clima de negocios en que todos pueden mejorar su desempeño, competitividad $y$ rentabilidad." (Cámara de Comercio de Medellín, 2010)

Fuente: Cámara de Comercio de Medellín.

Fuente: Elaboración propia. 
Ilustración 1. Composición Clúster de Construcción ${ }^{6}$

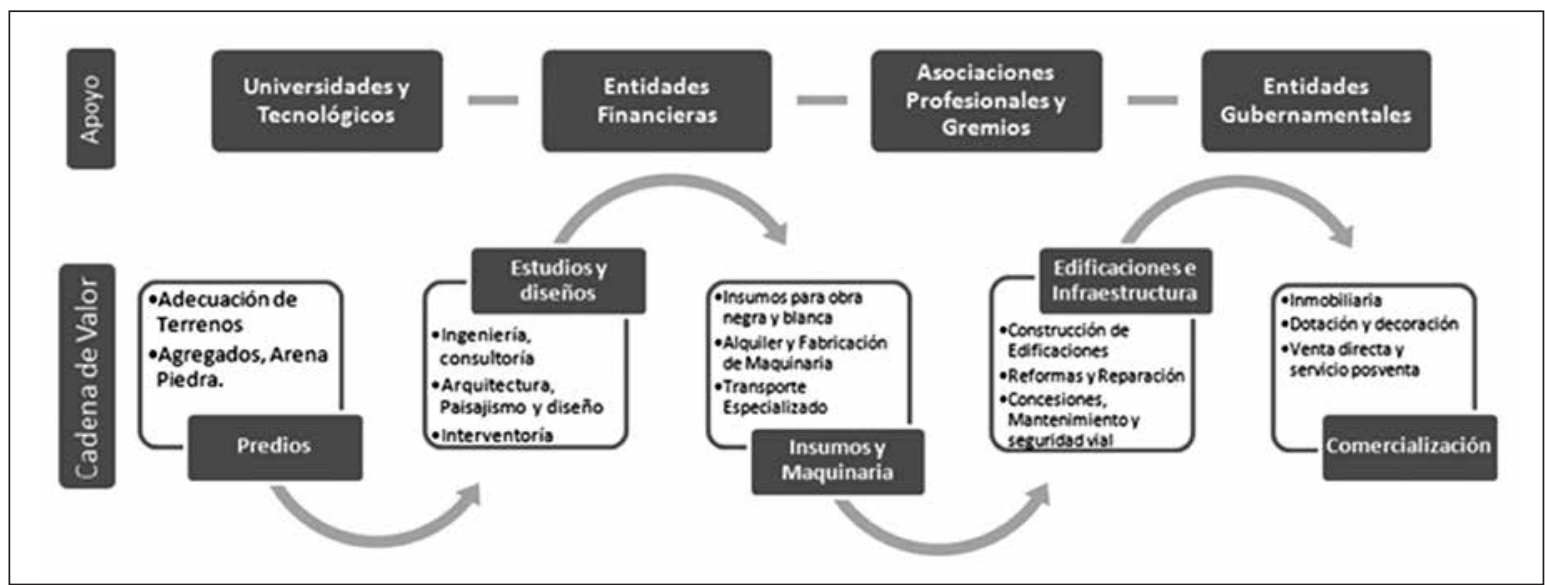

Los clúster se componen de empresas que forman parte de los eslabones de la cadena de valor, empezando con los proveedores de materias primas, siguiendo con los de transformación y al final con los de comercialización, apoyándose en otras para garantizar el buen funcionamiento de la cadena. Las empresas del clúster de Construcción fueron clasificadas en eslabones definidos por la Cámara de Comercio, los cuales son mostrados en Ilustración 1.

Correa, Castaño \& Ramírez (2010), proponen que un indicador financiero; es la relación existente entre dos variables de los estados financieros, los cuales permiten visualizar los puntos fuertes y débiles de las empresas y establecer probabilidades y tendencias; a continuación se describe el comportamiento de algunos indicadores financieros relacionados con la deuda y el patrimonio de las empresas objeto de estudio para entender el panorama financiero que presentaron en este periodo de tiempo; por tal motivo es importante realizar una lectura conjunta de sus resultados presentados en Anexo 1.

\subsubsection{Rentabilidad del patrimonio}

La rentabilidad del patrimonio, se expresa como el porcentaje de utilidad que los dueños de la empresa ganan sobre su inversión (Ortiz, 2008), este indicador se calcula:
Rentabilidad del patrimonio $=\frac{\text { Utilidad Neta }}{\text { Patrimonio Promedio }}$

Para las empresas del clúster construcción, el indicador presenta una disminución significativa en el periodo de crisis, reduciéndose de $13,09 \%$ a $8,12 \%$ en las empresas grandes y de $10,11 \%$ a $2,43 \%$ en las medianas, este comportamiento es debido a que la utilidad neta promedio disminuye en un porcentaje superior al aumento del patrimonio.

\subsubsection{Promedio Capital/Patrimonio}

Este indicador muestra el porcentaje del total del patrimonio que es representado por el capital aportado directamente por los socios de la empresa (Ortiz, 2008), y se calcula de la siguiente forma:

Promedio Capital / Patrimonio $=\frac{\text { Capital }}{\text { Patrimonio }}$

Para el clúster construcción, se observa una ligera tendencia a la baja debido al aumento del patrimonio a una tasa superior al del capital. Este comportamiento evidencia que en épocas de crisis no se realiza inyección de capital, sino que el incremento del patrimonio se origina a partir de cuentas como superávit y reservas.

6 Fuente: Elaboración propia con base en datos de la Cámara de Comercio de Medellín (2010) 


\subsubsection{Endeudamiento financiero}

Representa el porcentaje de las ventas de la empresa que es adeudado a las entidades financieras, (Ortiz, 2008), es decir, que implican un pago de intereses, el cálculo de este indicador se realiza así:

$$
\text { Endeudamiento financiero }=\frac{\text { Obligaciones financieras }}{\text { Ventas netas }}
$$

En las empresas medianas del clúster construcción, se presenta una reducción de la deuda financiera el período de crisis, en la mayoría de los casos coincide con una disminución del costo de la deuda en este período, mientras que las empresas grandes presentan una tendencia a incrementar la deuda en épocas de crisis y esto coincide con un aumento del costo de la deuda.

\subsubsection{Concentración del endeudamiento}

Representa el porcentaje de las obligaciones que deben ser cubiertas en el corto plazo (Ortiz, 2008), el cálculo de este indicador se realiza de la siguiente forma:

$$
\text { Concentración endeudamiento }=\frac{\text { Pasivo corriente }}{\text { Pasivo total }}
$$

En las empresas medianas, se evidencia una ligera tendencia a la baja en los años de crisis, donde el endeudamiento total y el de corto plazo disminuyen, mientras que las empresas grandes, muestran cierta estabilidad durante este período.

Martínez (2003) en el estudio Determinantes de la Fragilidad en las Empresas Colombianas, diseña un modelo para pronosticar fragilidad en las empresas colombianas clasificadas por sector económico, en donde existen variables de endeudamiento, rentabilidad y liquidez, con el fin de identificar rasgos de fragilidad. En el estudio se encontró que las empresas frágiles presentan mayores niveles de endeudamiento y menores niveles de rentabilidad y liquidez, adicionalmente, las razones financieras utilizadas son relevantes a la hora de pronosticar un deterioro en el estado de las empresas.

Como conclusión, la crisis económica afectó el promedio de las utilidades netas de las empresas de los diferentes clústeres, generando una disminución en la rentabilidad del patrimonio. Adicionalmente, el capital en los clústeres aumentó en una escala menor al crecimiento del patrimonio, para cuyos casos se encontró que las cuentas de superávit y reservas tuvieron incrementos sustanciales, mientras que la revalorización del patrimonio tuvo en términos generales una disminución. De otra parte, los indicadores que muestran el comportamiento de la deuda indican que las empresas tienden a aumentar la deuda financiera en épocas de crisis y la concentración de la deuda de corto plazo se encuentra en porcentajes altos, superiores al $75 \%$ del total del pasivo.

\subsection{Resultados}

Al realizar el estudio para las medianas y grandes empresas se pudo observar que la estructura en todos los años para el Cluster fue diferente de acuerdo al número de empresas que se anidó en cada quintil, sin embargo se observa una tendencia que la mayoría de las empresas tienen una estructura de deuda entre el rango del $0 \%$ y $60 \%$, mientras que unas pocas consideran el patrimonio como una fuente secundaria de financiación, puesto que existen empresas con un rango en la estructura entre el $80 \%$ y $100 \%$ representado en deuda. Este comportamiento se visualiza constante durante todos los años de estudio.

En cuanto al costo de la deuda, el comportamiento del clúster y tipo de empresa es diferente de acuerdo a su estructura de financiación. De manera general se observa en promedio que en la gran empresa el costo aumenta en la crisis y tiende a disminuir en épocas de auge, situación contraria en las empresas medianas que probablemente necesitan inyección de capital o liquidez via deuda, pero es necesario resaltar que a medida que el porcentaje de deuda aumenta en la estructura su costo disminuye.

Por otro lado, el costo del patrimonio en el clúster presenta comportamientos similares, entre los años 2005 - 2007 fue una tendencia creciente a pesar de entrar en época de crisis en el año 2007, sin embargo en el año 2008 en donde la crisis estuvo más acentuada se presenta una disminución considerable del $K a$, situación que se explica por una desvalorización del índice de mercado del país. 
El promedio del CPPC del clúster de construcción tiene una tendencia al alza durante todo el periodo de estudio, con la acotación que en el año 2008 se evidencia una disminución producto del comportamiento del costo del patrimonio dado que la estructura de capital tiene un peso ponderado más alto en este componente.

Se puede observar, que independientemente de la composición de la estructura financiera de las empresas expuestas en los primeros 4 quintiles, el costo de capital oscila en los mismos valores para las empresas grandes y medianas. En el primer caso el valor asciende a $9,58 \%$ y en el segundo es de $9,95 \%$, concluyendo que las empresas grandes tienen una mejor gestión de los recursos. Sin embargo, los valores promedio para el quintil 5 son valores menores a los presentados, pudiendo explicarse este suceso por el escudo tributario que tienen estas empresas al tener entre el $80 \%$ y $100 \%$ de su financiación en deuda.

\subsection{Discusión}

Para los cálculos del costo de patrimonio se utilizó el beta generado por las empresas colombianas referentes e inscritas en la Bolsa de Valores de Colombia, sin embargo se quizo realizar el ejercicio de calcular el beta de Damodaran para el clúster de construcción en Colombia, para lo cual se observa, en primera instancia, que en los años de crisis el beta para Damodaran es agresivo con un valor promedio de 1,21, mientras que en las empresas referentes en Colombia es defensivo con 0,9 , situación contraria al periodo de auge que manejan valores promedio de
0,87 y 1,04 respectivamente, sin embargo como se presenta en la tabla 3 , el $\beta_{\mathrm{L}}$ para ambos escenarios es muy similar pero si se calcula el componente del patrimonio con el beta de Damodaran es necesario incorporar al menos una prima por riesgo país lo cual incrementaría aún más el $\mathrm{Ka}$, condición que altera el cálculo del Ka levemente.

De acuerdo a los datos anteriores, se puede decir que si se toman los valores de las betas de Damodaran su comportamiento es acorde al riesgo del entorno puesto que en época de crisis las betas son más altas que en ciclos de auge en el clúster. Sin embargo, este comportamiento con los datos obtenidos en las empresas referentes de Colombia; es inversa a lo expuesto anteriormente en la tendencia de las betas, resultado que no es esperado por que a mayor riesgo evidenciado en la crisis, el beta debería ser más alta que en época de auge económico. Por tal razón se consideró mejor calcular el costo del patrimonio, teniendo en cuenta el beta de las empresas colombianas que tienen implícito los comportamientos del riesgo en el clúster.

\section{Conclusiones}

El componente de deuda no tiene un comportamiento claro en los ciclos económicos, sin embargo, presenta una tendencia creciente en el periodo de estudio, evidenciando un alza considerable en el año 2008; cuando la crisis fue generalizada en el ámbito mundial. Este efecto se puede explicar en primera instancia como una reacción del sector financiero ante la problemática del momento.

Tabla 3. Escenarios de cálculo de Betas ${ }^{7}$

\begin{tabular}{|c|c|c|c|c|c|c|}
\hline Años & $\begin{array}{c}\text { Bu } \\
\text { Colombia }\end{array}$ & $\begin{array}{c}\text { Bu } \\
\text { Damodaran }\end{array}$ & Impuestos & D/P & $\begin{array}{c}\text { BI } \\
\text { Colombia }\end{array}$ & $\begin{array}{c}\text { BI } \\
\text { Damodaran }\end{array}$ \\
\hline 2005 & 0,96 & 0,80 & $35,0 \%$ & $39,0 \%$ & 1,20 & 1,00 \\
\hline 2006 & 1,12 & 0,95 & $35,0 \%$ & $44,3 \%$ & 1,44 & 1,22 \\
\hline 2007 & 0,85 & 1,09 & $34,0 \%$ & $42,0 \%$ & 1,09 & 1,39 \\
\hline 2008 & 0,80 & 1,22 & $33,0 \%$ & $41,7 \%$ & 1,02 & 1,56 \\
\hline 2009 & 1,05 & 1,20 & $33,0 \%$ & $41,3 \%$ & 1,35 & 1,54 \\
\hline
\end{tabular}

7 Fuente: Elaboración Propia. 
El componente del patrimonio en los ciclos económicos contemplados no tiene una tendencia marcada, solamente se observa un crecimiento moderado desde el año 2005, pero se destaca que en el año 2008 hubo una disminución del valor en el clúster Construcción debido a la variable $R_{m}$, lo que permite cuestionar la metodología de cálculo del Ka para mercados emergentes no diversificados con empresas que no cotizan en la bolsa de valores, dado que actualmente la única alternativa teórica existente es el CAPM y sus modificaciones con primas de riesgo adicionales como riesgo país, riesgo por tamaño, entre otras.

Las empresas no reaccionan de la misma forma ante las épocas de crisis ya que su comportamiento depende en gran medida de proyectos en desarrollo, conexión con los mercados externos, inversiones en moneda extranjera, etc., lo cual se evidencia en la recuperación del año 2009 en su componente de patrimonio y de la composición de la estructura financiera.

Desde el punto de vista financiero se espera que $K_{a}$ $>K_{d}(1-t)$, sin embargo, en el clúster Construcción se observó que este supuesto no se cumple en la mitad de los casos del periodo de estudio, lo cual puede explicarse por la estructura de capital y la gestión de los componentes de deuda y patrimonio.

El costo de capital tiene el mismo comportamiento del componente de patrimonio debido al peso ponderado que tiene esta variable en la estructura de capital, sin embargo, se considera que el costo de operación de las empresas en los diferentes ciclos económicos fue estable y adecuado con respecto a las variables macroeconómicas.

Es de reconocer que la actual información que dispone el país para el análisis presentado, amerita que se avance en la construcción de sistemas de información eficientes para la captura de la información financiera de las empresas, sin importar la entidad que supervise (Cámaras de Comercio, las Superintendencias, entre otras insituciones), dado que la información disponible actualmente presenta algunos inconvenientes que afectan la calidad de la misma y por ende no permiten hacer análisis detallados.
Los resultados obtenidos pueden servir como base a futuras investigaciones que se interesen por el análisis y la toma de decisiones de la empresa a partir de los componentes de la estructura de capital y el costo de capital, por medio del diseño de una metodología para el cálculo del costo del patrimonio aplicable a las empresas que no coticen en la bolsa de valores de mercados emergentes, variable fundamental para el CPPC.

\section{Referencias}

Cámara de Comercio de Medellín. (2010). Clúster de Construcción. Extraído el 11 de Julio de 2010, de: http://www.camaramed. org.co/construccion/

Comisión de Regulación de Energía y Gas (2002). Costo Promedio de Capital: Metodología de Cálculo para la Distribución de Energía Eléctrica y Gas Combustible por Redes. Documento CREG, 022.

Cruz, S., Villareal, J. \& Rosillo, J. (2003). Finanzas corporativas. Valoración, política de financiamiento y riesgo. Teoría \& Práctica. Bogotá: Thomson.

Correa, J., Castaño, C. \& Ramírez, L. (2010). Análisis Financiero Integral: elementos para el desarrollo de las organizaciones. En: Lúmina, 12: 180-193.

Damodaran, A. (2002). Investment Valuation. Segunda Edición. Editorial John Wiley \& Sons, New York.

Decreto 2650 de 1993. Plan Único de Cuentas. Superintendencia de Sociedades. Extraído el 03 de Junio de 2010, de: http:// www.supersociedades.gov.co

Emery, D., \& Finnerty, J. (2000). Fundamentos de Administración Financiera. Pearson Education.

Fernández, V. (2005). El modelo CAPM para distintos horizontes de tiempo. En: Revista Ingeniería de Sistemas, Vol. XIX: 7-18.

García, O. (2003). Valoración, Gerencia del Valor y EVA. Cali: Prensa Moderna.

García, O. (2009). Administración Financiera, Fundamentos y Aplicaciones. Cali: Prensa Moderna.

Gitman, L. (2007). Principios de Administración Financiera. Edición $11^{\circ}$. Pearson Educación. México.

Grinblatt, M. \& Titman, L. (2003). Mercados financieros y estrategia empresarial. - 2. ed. España: McGraw-Hill

Jaramillo, F. (2010). Principios de Valoración de Empresas. Medellín: ECOE.

Ley 590 de 2000. Por la cual se dictan disposiciones para promover el desarrollo de las micro, pequeñas y medianas empresa. Congreso de Colombia. Disponible en: http://www.secretariasenado.gov. co/senado/basedoc/ley/2000/ley_0590_2000.html

Ley 905 de 2004. Por medio de la cual se modifica la Ley 590 de 2000 sobre promoción del desarrollo de las micro, pequeña y mediana empresa colombiana y se dictan otras disposiciones. Congreso de Colombia. Disponible en: http://www.secretariasenado.gov.co/senado/basedoc/ley/2004/ley_0905_2004.html

Lintner, J. (1965). The Valuation of Risk Assets and the Selection of Risky Investment in Stock Portfolios and Capital Budgets. En: The Review of Economics and Statistics , 47 (1): 13-37. 
Martínez, A. (2003). Determinantes de fragilidad en las empresas colombianas. Borradores de Economía, Banco de la Republica. Disponible en: http://www.banrep.org/docum/ftp/borra259.pdf

Mejía, L. (2000). Costo de Capital Promedio Ponderado. Extraido el 14 de Septiembre de 2010, de: ftp://ftp.eia.edu.co/Academico/ Postgrado/.../ccpp\%20instructivo.doc

Mossin, J. (1966). Equilibrium in a Capital Asset Market. En: Econométrica, 34 (4): 768-783.

Ortiz, H. (2008). Análisis financiero aplicado y principios de administración financiera (13 ed.). Bogotá: Universidad Externado de Colombia.
Ross, S. Westerfield, R. \& Jordan, B. (2001). Fundamentos de Finanzas Corporativas. Quinta Edición. McGraw Hill Interamericana Editores S.A. México.

Vélez, I. (2003). El costo promedio de capital. Politécnico Grancolombiano. Extraído el 18 de Mayo de 2010, de http://sigma. poligran.edu.co/politecnico/apoyo/Decisiones/curso/Elcostodecapitalppt.pdf

Villareal, J. (2005). El costo de capital en proyectos de infraestructura civil básica (IB): Un ejemplo práctico: el WACC para una concesión aeroportuaria. En: Revista de Ingeniería, Facultad de Ingeniería Universidad de los Andes, 21: 19-29. 


\section{ANEXO 1}

Resultados indicadores financieros ${ }^{8}$

\begin{tabular}{|c|c|c|c|c|}
\cline { 2 - 5 } \multicolumn{1}{c|}{} & \multicolumn{2}{c|}{} & \multicolumn{2}{c|}{$\begin{array}{c}\text { Rentabilidad del } \\
\text { Capital / Patrimonio }\end{array}$} \\
\hline Año & Grande & Mediana & Grande & Mediana \\
\hline 2005 & $15,1 \%$ & $26,3 \%$ & & \\
\hline 2006 & $13,5 \%$ & $18,5 \%$ & $10,08 \%$ & $8,32 \%$ \\
\hline 2007 & $11,7 \%$ & $16,9 \%$ & $13,09 \%$ & $10,11 \%$ \\
\hline 2008 & $11,4 \%$ & $16,1 \%$ & $11,12 \%$ & $6,46 \%$ \\
\hline 2009 & $10,0 \%$ & $16,1 \%$ & $8,12 \%$ & $2,43 \%$ \\
\hline
\end{tabular}

\begin{tabular}{|c|c|c|c|c|}
\cline { 2 - 5 } \multicolumn{1}{c|}{} & $\begin{array}{c}\text { Nivel de Endeudamiento } \\
\text { Financiero }\end{array}$ & \multicolumn{2}{c|}{$\begin{array}{c}\text { Concentración del } \\
\text { Endeudamiento }\end{array}$} \\
\hline Año & Grande & Mediana & Grande & Mediana \\
\hline 2005 & $24,36 \%$ & $24,25 \%$ & $76,99 \%$ & $84,79 \%$ \\
\hline 2006 & $24,04 \%$ & $27,64 \%$ & $76,58 \%$ & $82,71 \%$ \\
\hline 2007 & $28,02 \%$ & $27,15 \%$ & $79,45 \%$ & $88,43 \%$ \\
\hline 2008 & $29,29 \%$ & $25,11 \%$ & $82,75 \%$ & $87,03 \%$ \\
\hline 2009 & $29,48 \%$ & $24,13 \%$ & $78.75 \%$ & $85.23 \%$ \\
\hline
\end{tabular}

8 Fuente: Elaboración Propia. 


\section{ANEXO 2}

Resultados componentes costo de capital ${ }^{9}$

\begin{tabular}{|c|c|c|c|c|c|c|c|c|c|c|c|c|c|c|}
\hline \multirow[b]{2}{*}{ Quintil } & \multirow[b]{2}{*}{ Año } & \multicolumn{2}{|c|}{$K d^{*}(1-t)$} & \multirow[b]{2}{*}{$\mathbf{t}$} & \multirow[b]{2}{*}{$\mathbf{R m}$} & \multirow[b]{2}{*}{ Rf } & \multicolumn{2}{|c|}{ BI } & \multicolumn{2}{|c|}{ Ka } & \multicolumn{2}{|c|}{ WACC } & \multicolumn{2}{|c|}{ Empresas } \\
\hline & & Grande & Mediana & & & & Grande & Mediana & Grande & Mediana & Grande & Mediana & Grande & Mediana \\
\hline \multirow[t]{5}{*}{1} & 2005 & $16,8 \%$ & $9,8 \%$ & $35 \%$ & $6,1 \%$ & $6,0 \%$ & 1,06 & 0,97 & $6,1 \%$ & $6,1 \%$ & $7,53 \%$ & $6,11 \%$ & 29 & 6 \\
\hline & 2006 & $14,7 \%$ & $23,3 \%$ & $35 \%$ & $9,8 \%$ & $6,9 \%$ & 1,24 & 1,16 & $10,6 \%$ & $10,3 \%$ & $11,14 \%$ & $11,01 \%$ & 25 & 10 \\
\hline & 2007 & $18,8 \%$ & $18,3 \%$ & $34 \%$ & $10,7 \%$ & $8,6 \%$ & 0,94 & 0,87 & $10,5 \%$ & $10,4 \%$ & $11,65 \%$ & $10,67 \%$ & 27 & 9 \\
\hline & 2008 & $16,4 \%$ & $13,9 \%$ & $33 \%$ & $8,9 \%$ & $9,8 \%$ & 0,87 & 0,83 & $9,0 \%$ & $9,0 \%$ & $9,92 \%$ & $9,35 \%$ & 29 & 14 \\
\hline & 2009 & $15,5 \%$ & $19,7 \%$ & $33 \%$ & $9,6 \%$ & $5,9 \%$ & 1,16 & 1,11 & $10,2 \%$ & $10,0 \%$ & $10,90 \%$ & $10,79 \%$ & 30 & 13 \\
\hline \multirow[t]{5}{*}{2} & 2005 & $10,0 \%$ & $11,0 \%$ & $35 \%$ & $6,1 \%$ & $6,0 \%$ & 1,19 & 1,24 & $6,1 \%$ & $6,1 \%$ & $7,14 \%$ & $7,60 \%$ & 15 & 14 \\
\hline & 2006 & $11,3 \%$ & $11,2 \%$ & $35 \%$ & $9,8 \%$ & $6,9 \%$ & 1,39 & 1,43 & $11,0 \%$ & $11,1 \%$ & $11,07 \%$ & $11,15 \%$ & 16 & 10 \\
\hline & 2007 & $11,6 \%$ & $13,7 \%$ & $34 \%$ & $10,7 \%$ & $8,6 \%$ & 1,08 & 1,11 & $10,8 \%$ & $10,9 \%$ & $11,04 \%$ & $11,77 \%$ & 18 & 12 \\
\hline & 2008 & $17,1 \%$ & $10,2 \%$ & $33 \%$ & $8,9 \%$ & $9,8 \%$ & 1,00 & 1,04 & $8,9 \%$ & $8,9 \%$ & $11,12 \%$ & $9,27 \%$ & 13 & 8 \\
\hline & 2009 & $16,3 \%$ & $11,3 \%$ & $33 \%$ & $9,6 \%$ & $5,9 \%$ & 1,34 & 1,35 & $10,9 \%$ & $10,9 \%$ & $12,45 \%$ & $11,01 \%$ & 11 & 10 \\
\hline \multirow[t]{5}{*}{3} & 2005 & $6,4 \%$ & $9,6 \%$ & $35 \%$ & $6,1 \%$ & $6,0 \%$ & 1,63 & 1,51 & $6,1 \%$ & $6,1 \%$ & $6,26 \%$ & $7,73 \%$ & 13 & 14 \\
\hline & 2006 & $8,6 \%$ & $10,1 \%$ & $35 \%$ & $9,8 \%$ & $6,9 \%$ & 1,82 & 1,78 & $12,3 \%$ & $12,2 \%$ & $10,46 \%$ & $11,19 \%$ & 13 & 9 \\
\hline & 2007 & $10,8 \%$ & $10,8 \%$ & $34 \%$ & $10,7 \%$ & $8,6 \%$ & 1,37 & 1,43 & $11,4 \%$ & $11,6 \%$ & $11,10 \%$ & $11,17 \%$ & 10 & 11 \\
\hline & 2008 & $10,9 \%$ & $13,2 \%$ & $33 \%$ & \begin{tabular}{l|l}
$8,9 \%$ & \\
\end{tabular} & $9,8 \%$ & 1,39 & 1,34 & $8,6 \%$ & $8,6 \%$ & $9,81 \%$ & $10,93 \%$ & 12 & 13 \\
\hline & 2009 & $9,7 \%$ & $9,2 \%$ & $33 \%$ & $9,6 \%$ & $5,9 \%$ & 1,86 & 1,75 & $12,8 \%$ & $12,4 \%$ & $11,14 \%$ & $10,80 \%$ & 18 & 12 \\
\hline \multirow[t]{5}{*}{4} & 2005 & $4,7 \%$ & $1,3 \%$ & $35 \%$ & $6,1 \%$ & $6,0 \%$ & 2,20 & 2,22 & $6,1 \%$ & $6,1 \%$ & $5,17 \%$ & $2,89 \%$ & 7 & 1 \\
\hline & 2006 & $5,9 \%$ & $8,3 \%$ & $35 \%$ & $9,8 \%$ & $6,9 \%$ & 2,63 & 2,45 & $14,7 \%$ & $14,2 \%$ & $8,73 \%$ & $10,36 \%$ & 10 & 7 \\
\hline & 2007 & $6,6 \%$ & $5,8 \%$ & $34 \%$ & $10,7 \%$ & $8,6 \%$ & 2,05 & 1,96 & $12,8 \%$ & $12,6 \%$ & $8,58 \%$ & $8,13 \%$ & 9 & 4 \\
\hline & 2008 & $8,2 \%$ & $20,8 \%$ & $33 \%$ & $8,9 \%$ & $9,8 \%$ & 1,83 & 2,24 & $8,2 \%$ & $7,9 \%$ & $8,19 \%$ & $17,33 \%$ & 11 & 2 \\
\hline & 2009 & $4,7 \%$ & & $33 \%$ & $9,6 \%$ & $5,9 \%$ & 2,49 & & $15,1 \%$ & & $8,17 \%$ & & 7 & \\
\hline \multirow[t]{5}{*}{5} & 2005 & $2,5 \%$ & $11,1 \%$ & $35 \%$ & $6,1 \%$ & $6,0 \%$ & 7,47 & 6,83 & $6,4 \%$ & $6,4 \%$ & $2,85 \%$ & $10,62 \%$ & 5 & 3 \\
\hline & 2006 & $4,0 \%$ & $7,9 \%$ & $35 \%$ & $9,8 \%$ & $6,9 \%$ & 8,36 & 12,33 & $31,7 \%$ & $43,5 \%$ & $6,57 \%$ & $10,12 \%$ & 5 & 2 \\
\hline & 2007 & $4,2 \%$ & $10,2 \%$ & $34 \%$ & $10,7 \%$ & $8,6 \%$ & 7,08 & 9,09 & $23,1 \%$ & $27,2 \%$ & $5,78 \%$ & $11,30 \%$ & 5 & 2 \\
\hline & 2008 & $3,2 \%$ & $0,1 \%$ & $33 \%$ & $8,9 \%$ & $9,8 \%$ & 8,10 & 27,41 & $2,9 \%$ & $-13,3 \%$ & $3,20 \%$ & $-0,14 \%$ & 4 & 1 \\
\hline & 2009 & $3,3 \%$ & $8,9 \%$ & $33 \%$ & $9,6 \%$ & $5,9 \%$ & 10,83 & 7,60 & $46,1 \%$ & $34,1 \%$ & $6,19 \%$ & $11,37 \%$ & 3 & 3 \\
\hline
\end{tabular}

\footnotetext{
9 Fuente: Elaboración Propia.
} 